\title{
A Relation between Verkley's 1987 Modon and 1990 Modon
}

\author{
by \\ Hirotada Kanehisa \\ Meteorological Research Institute, Tsukuba, Ibaraki, 305 Japan
}

(Received January 25, 1991 ; Revised August 18, 1992)

\begin{abstract}
Verkley $(1987,1990)$ constructed modons as a model of the atmospheric blocking phenomenon. They are exact solutions of the barotropic potential vorticity equation on a sphere. In this note, it is shown that the 1987 modon changes continuously into the 1990 modon as the value of $-d Q / d \Psi$ within the modon goes to zero, where $Q$ and $\Psi$ are potential vorticity and streamfunction of the 1987 modon, respectively. Thereby it is revealed that the stability properties of the 1990 modon can be studied by means of Arnol'd's invariant of the 1987 modon though Arnol'd's invariant can not be defined for the 1990 modon.
\end{abstract}

\section{Introduction}

Atmospheric blocking patterns may be modeled by stable equilibrium points of the fluid dynamical system because of their quasistationarity and longevity. Since the vertical structure of atmospheric blocking is equivalent barotropic, we may take a barotropic dynamical system as a first approximation.

Modons by Larichev and Reznik (1976), which are solitary wave solutions of the potential vorticity equation on a $\beta$-plane, have the same spatial pattern as atmospheric blocking, and many researchers (e.g., McWilliams, 1980 ; Haines and Marshall, 1987; Butchart et al., 1989) discussed modons as a model of the atmospheric blocking phenomenon.

Modons on a $\beta$-plane may not be a good model of blocking which is of planetary scale. Tribbia (1984) and Verkley (1984, 1987, 1990) constructed modons on a sphere. They have a

(C) 1992 by the Meteorological Research Institute stronger anticyclone to the north of a weaker cyclone, in contrast to modons on a $\beta$-plane which have no such asymmetry. Modons on a sphere bear greater resemblance to atmospheric blocking.

As for Verkley $(1987,1990)$ 's modons (hereafter referred to as the 87 modon and the 90 modon, respectively), the flow pattern outside the dipolar blocking region is wavy in contrast to Verkley's 1987 and Tribbia's 1984 modons, for which the flow pattern is essentially zonal there.

Potential vorticity $Q$ and streamfunction $\Psi$ of modon are functionally related, because modon is a stationary (or longitudinally propagating) solution of the barotropic potential vorticity equation. Two functional relationships are specified inside and outside the blocking region. For the 87 modon, $Q$ is assumed to be a linear function of $\Psi$ inside the blocking region, while for 90 modon $Q$ is constant there. In both modons, $Q$ is a linear 
function of $\Psi$ outside the blocking region.

Verkley $(1987,1990)$ tried to prove the linear stability of the 87 and 90 modons. In his papers, the exponential growth rate of disturbances was numerically calculated. However, the non-positiveness of the growth rate was not demonstrated.

It is well known that for any disturbance $\phi$ added to a stationary basic state $\Psi$ there exists a conserved quantity $A[\phi]$ called Arnol'd's invariant which is $O\left(\phi^{2}\right)$ as $\phi \rightarrow 0$ (see, e.g., McIntyre and Shepherd, 1987). This invariant $A[\phi]$ is a useful tool in studying the stability properties of the basic state $\Psi$ analytically (see, e.g., Benzi et al., 1982 ; Laedke and Spatschek, 1986), even when the normal mode approach can not be applied to this state analytically. For Arnol'd's invariant $A[\phi]$ to exist, $d \Psi / d Q$ must be well defined in the entire region. For the 87 modon, $d \Psi / d Q$ is well defined because $\Psi$ is a linear function of $Q$ and so Arnol'd's invariant $A[\phi]$ exists. It is not defined inside the blocking region of the 90 modon, which makes it difficult to apply Arnol'd's method in this case.

In this note, it is shown that the 87 modon changes continuously into the 90 modon as a positive parameter $k^{2}$ goes to zero. This parameter is the value of $-d Q / d \Psi$ inside the blocking region. Thereby it is revealed that the stability properties of the 90 modon can be studied by means of Arnol'd's invariant of the 87 modon for a sufficiently small $k^{2}$ and therefore that the above mentioned difficulty for the 90 modon can be overcome.

The organization of this note is as follows. In section 2 , a brief outline of the construction of the 87 and 90 modons is given. In section 3 , the relation between the two modons is investigated. In section 4, stability properties are considered. Concluding remarks are presented in Section 5.

\section{The 87 modon and the 90 modon}

The 87 and 90 modons of Verkley are stationary (or longitudinally propagating) solutions to the barotropic potential vorticity equation on a sphere,

$$
\partial Q / \partial t+\mathrm{J}(\Psi, Q)=0,
$$

where $Q=\Delta \Psi+2 \Omega \mu$.

In the spherical coordinate system, Jacobian $\mathrm{J}($, ) and Laplacian $\Delta$ are given by

$$
\begin{gathered}
\mathrm{J}(A, B)=\left(1 / R^{2}\right)\{(\partial A / \partial \lambda)(\partial B / \partial \mu) \\
-(\partial B / \partial \lambda)(\partial A / \partial \mu)\} \\
\Delta=\left(1 / R^{2}\right)\left\{(\partial / \partial \mu)\left(1-\mu^{2}\right)(\partial / \partial \mu)\right. \\
\left.+\left(1 / 1-\mu^{2}\right)\left(\partial^{2} / \partial \lambda^{2}\right)\right\} .
\end{gathered}
$$

Here $\Psi$ is the streamfunction, $Q$ the potential vorticity, $\Omega$ the angular velocity of the earth, $R$ the radius of the earth and $\lambda$ longitude, $\theta$ latitude, $\mu=\sin \theta$ and $t$ time. The streamfunction $\Psi$ is decomposed as follows:

$$
\Psi=\Phi-R U \mu+C,
$$

where $-R U \mu$ represents a solid body rotation and $C$ is a constant. By this decomposition, Eq. (1) is rewritten as

$$
\begin{aligned}
& \partial Q / \partial t \\
& \quad+\mathrm{J}(\Phi-R U \mu+C, \Delta \Phi+\{(2 U / R)+2 \Omega\} \mu) \\
& \quad=0 .
\end{aligned}
$$

For a stationary solution (for a propagating solution, the following argument is essentially unchanged) Eq. (3) becomes

$$
\mathrm{J}(\Phi-R U \mu+C, \Delta \Phi+\{(2 U / R)+2 \Omega\} \mu)=0 .
$$

Equation (4) implies that the potential vorticity $Q=\Delta \Phi+\{(2 U / R)+2 \Omega\} \mu$ is a function of the streamfunction $\Psi=\Phi-R U \mu+C$,

$$
Q=F(\Psi) .
$$

Let a point $(\lambda, \theta)=\left(\lambda_{0}, \theta_{0}\right)$ be the centre of modons, and consider such a coordinate transformation $(\lambda, \theta) \rightarrow\left(\lambda^{\prime}, \theta^{\prime}\right)$ that this point $(\lambda, \theta)$ $=\left(\lambda_{0}, \theta_{0}\right)$ in the old coordinate system is transformed into the north pole $\theta^{\prime}=\pi / 2$ in the new one (see Fig. 1). Under this transformation, the variable $\mu=\sin \theta$ in the old coordinate sys- 




Fig. 1 Coordinate transformation $(\lambda, \theta) \rightarrow\left(\lambda^{\prime}, \theta^{\prime}\right)$.

tem is represented in terms of the new coordinates $\lambda^{\prime}, \theta^{\prime}$ as

$$
\begin{aligned}
\mu & =\sin \theta \\
& =\sin \theta_{0} \sin \theta^{\prime}-\cos \theta_{0} \cos \theta^{\prime} \cos \lambda^{\prime}
\end{aligned}
$$

In this coordinate transformation, Laplacian $\Delta$ and Jacobian $\mathrm{J}(A, B)$ do not change their forms. Hereafter the primes of new coordinates $\lambda^{\prime}, \theta^{\prime}$ are dropped.

By a latitude circle $\left\{(\lambda, \theta): \theta=\theta_{a}=\right.$ constant\}, the surface of the sphere are partitioned into two regions as shown in Fig. 2. Here $\left\{(\lambda \leqq \theta): \pi / 2 \geqq \theta>\theta_{a}\right\}$ is called the inner region (hereafter denoted by subscript in) and



Fig. 2 Inner region and outer region.

$\left\{(\lambda, \theta): \theta_{a}>\theta \geqq-\pi / 2\right\}$ is called the outer region (hereafter denoted by subscript ex). For the 87 modon, the function $Q=F(\Psi)$ is assumed to have the following form:

$$
\begin{aligned}
& Q_{\text {in }}=-k^{2}\left(\Psi_{\text {in }}-C_{\text {in }}\right) \\
& \text { for } \pi / 2 \geqq \theta>\theta_{a},
\end{aligned}
$$

and

$$
\begin{aligned}
Q_{\mathrm{ex}}=-p^{2}\left(\Psi_{\mathrm{ex}}-C_{\mathrm{ex}}\right) \\
\quad \text { for } \theta_{a}>\theta \geqq-\pi / 2,
\end{aligned}
$$

where $k^{2}$ and $p^{2}$ are positive constants. Eqs. (7) and (8) are second order differential equations for $\Psi$ with the boundary conditions that $\Psi_{\text {in }}$ and $\Psi_{\text {ex }}$ are connected continuously up to the second derivative on the circle $\left\{(\lambda, \theta): \theta=\theta_{a}\right\}$. The solution is given by

$$
\begin{aligned}
& \Psi_{\text {in }}=C_{\text {in }}-R U_{\text {in }} \sin \theta_{0} \cos \theta_{a}\left\{P_{\alpha}^{0}(\sin \theta) / P^{1}{ }_{\alpha}\left(\sin \theta_{a}\right)+\sin \theta / \cos \theta_{a}\right\} \\
& -R U_{\text {in }} \cos \theta_{0} \cos \theta_{a}\left\{P_{\alpha}^{1}(\sin \theta) / P_{\alpha}^{1}\left(\sin \theta_{a}\right)-\cos \theta / \cos \theta_{a}\right\} \cos \lambda \\
& =\Psi^{M}{ }_{\text {in }}(\theta)+\Psi^{D}{ }_{\text {in }}(\theta) \cos \lambda
\end{aligned}
$$

and

$$
\begin{aligned}
\Psi_{\mathrm{ex}}= & C_{\mathrm{ex}}+R U_{\mathrm{ex}} \sin \theta_{0} \cos \theta_{a}\left\{P_{\beta}^{0}(-\sin \theta) / P^{1}{ }_{\beta}\left(-\sin \theta_{a}\right)-\sin \theta / \cos \theta_{a}\right\} \\
& \quad-R U_{\mathrm{ex}} \cos \theta_{0} \cos \theta_{a}\left\{P^{1}{ }_{\beta}(-\sin \theta) / P^{1}{ }_{\beta}\left(-\sin \theta_{a}\right)-\cos \theta / \cos \theta_{a}\right\} \cos \lambda \\
= & \Psi^{M}{ }_{\mathrm{ex}}(\theta)+\Psi^{D}{ }_{\mathrm{ex}}(\theta) \cos \lambda,
\end{aligned}
$$


where $P^{n}{ }_{\nu}(X)$ are associated Legendre functions of the first kind with degree $\nu$ and order $n$, and the following equations must be satisfied:

$$
\begin{aligned}
& \alpha(\alpha+1)=2\left\{\left(R \Omega / U_{\mathrm{in}}\right)+1\right\}=R^{2} k^{2}, \\
& \alpha>-1 / 2, \\
& \beta(\beta+1)=2\left\{\left(R \Omega / U_{\mathrm{ex}}\right)+1\right\}=R^{2} p^{2}, \\
& \beta>-1 / 2,
\end{aligned}
$$

and

$$
\begin{aligned}
& \left(1 / U_{\mathrm{ex}}\right) P_{\beta}^{1}\left(-\sin \theta_{a}\right) / P^{2}{ }_{\beta}\left(-\sin \theta_{a}\right) \\
& =-\left(1 / U_{\mathrm{in}}\right) P^{1}{ }_{\alpha}\left(\sin \theta_{a}\right) / P^{2}{ }_{\alpha}\left(\sin \theta_{a}\right) .
\end{aligned}
$$

Although associated Legendre functions $P^{n}{ }_{\nu}(X)$ are not finite at $X=-1$ for nonintegers $\nu$, there are no problems in Eqs. (9) and (10) since $P^{n}{ }_{\nu}(\sin \theta)$ and $P^{n}{ }_{\nu}(-\sin \theta)$ are defined in the inner and outer regions respectively. In Eqs. (9) and (10), superscripts $M$ and $D$ denote the monopole and dipole components of $\Psi$, respectively. If $\theta_{0}$ and $\theta_{a}$ which represent the position and extention of a blocking region and $U_{\text {ex }}$ which represents the magnitude of westerly wind are prescribed, then $\beta(\beta+1)$ is determined by Eq. (12), and then the value of the left hand side of Eq. (13) is given. Finally $\alpha(\alpha+1)$ and $U_{\text {in }}$ are determined by Eqs. (11) and (13). The value of $C_{\text {ex }}$ can be assumed to be zero without loss of generality, and the value of $C_{\mathrm{in}}$ is determined by the boundary conditions.

On the other hand, for the 90 modon the function $Q=F(\Psi)$ is assumed to have the following form:

$$
Q_{\text {in }}=\bar{Q}_{\text {in }}=\text { constant }
$$

$$
\text { for } \pi / 2 \geqq \theta>\theta_{a},
$$

and

$$
\begin{aligned}
& Q_{\mathrm{ex}}=-p^{2}\left(\Psi_{\mathrm{ex}}-C_{\mathrm{ex}}\right) \\
& \text { for } \theta_{a}>\theta \geqq-\pi / 2,
\end{aligned}
$$

where $p^{2}$ is a positive constant. Eqs. (14) and (15) are second order differential equations for $\Psi$ with the boundary conditions that $\Psi_{\text {in }}$ and $\Psi_{\text {ex }}$ are continuously connected up to the second derivatives on the circle $\left\{(\lambda, \theta): \theta=\theta_{a}\right\}$. The solution is given by

$$
\begin{aligned}
\Psi_{\text {in }}= & C_{\text {in }}-R^{2} \Omega \sin \theta_{0} \cos \theta_{a}\left\{2 \log \cos (\pi / 4-\theta / 2) / \tan \left(\pi / 4-\theta_{a} / 2\right)-\sin \theta / \cos \theta_{a}\right\} \\
& \quad+R^{2} \Omega \cos \theta_{0} \cos \theta_{a}\left\{\tan (\pi / 4-\theta / 2) / \tan \left(\pi / 4-\theta_{a} / 2\right)-\cos \theta / \cos \theta_{a}\right\} \cos \lambda \\
= & \Psi^{M}{ }_{\text {in }}(\theta)+\Psi^{D}{ }_{\text {in }}(\theta) \cos \lambda,
\end{aligned}
$$

and

$$
\begin{aligned}
\Psi_{\mathrm{ex}}= & C_{\mathrm{ex}}+R U_{\mathrm{ex}} \sin \theta_{0} \cos \theta_{a}\left\{P^{0}{ }_{\beta}(-\sin \theta) / P^{1}{ }_{\beta}\left(-\sin \theta_{a}\right)-\sin \theta / \cos \theta_{a}\right\} \\
& \quad-R U_{\mathrm{ex}} \cos \theta_{0} \cos \theta_{a}\left\{P^{1}{ }_{\beta}(-\sin \theta) / P^{1}{ }_{\beta}\left(-\sin \theta_{a}\right)-\cos \theta / \cos \theta_{a}\right\} \cos \lambda \\
= & \Psi^{M}{ }_{\mathrm{ex}}(\theta)+\Psi^{D}{ }_{\mathrm{ex}}(\theta) \cos \lambda
\end{aligned}
$$

where the following equations must be satisfied:

$$
\begin{aligned}
& \bar{Q}_{\mathrm{ln}}=\Omega \sin \theta_{0} \cos \theta_{a} / \tan \left(\pi / 4-\theta_{a} / 2\right), \\
& \beta(\beta+1)=2\left\{\left(R \Omega / U_{\mathrm{ex}}\right)+1\right\}=R^{2} p^{2}, \\
& \beta>-1 / 2,
\end{aligned}
$$

and

$$
\begin{aligned}
& \left(1 / U_{\mathrm{ex}}\right) P^{1}{ }_{\beta}\left(-\sin \theta_{a}\right) / P^{2}{ }_{\beta}\left(-\sin \theta_{a}\right) \\
& =(R \Omega)^{-1} \cot \left(\pi / 4-\theta_{a} / 2\right) .
\end{aligned}
$$

If $\theta_{0}$ representing the position of a blocking region and $U_{\text {ex }}$ representing the magnitude of 
westerly wind are prescribed, then $\beta(\beta+1)$ is determined by Eq. (19), and then $\theta_{a}$ is determined by Eq. (20). Finally the value of $\bar{Q}_{\text {in }}$ is given by Eq. (18). The value of $C_{\text {ex }}$ can be assumed to be zero without loss of generality, and the value of $C_{\text {in }}$ is determined by the boundary conditions.

\section{The 90 modon as a limiting case of the 87 modon}

By a glimpse at Eqs. (7) and (14), one can anticipate that the 87 modon changes into the 90 modon as $k^{2}$ goes to zero. In this section it is shown that this anticipation is indeed true though the differential equations (7) and (14) are of different type from each other.

From Eq. (11), it is easily seen that $\alpha$ goes to zero as $k^{2}$ goes to zero. In order to consider a limiting case $\alpha \rightarrow 0$ of the 87 modon, the limit $\alpha \rightarrow 0$ of associated Legendre functions $P^{m}{ }_{\alpha}(X)$ must be calculated. Associated Legendre functions (e.g., see Appendix in Verkley, 1984) are expressed as

$$
\begin{aligned}
& P_{\alpha}^{m}(X)=P_{\alpha}^{-m}(X)(-1)^{m} \Gamma(\alpha+m+1) / \Gamma(\alpha-m+1) \\
& P_{\alpha}^{-m}(X)=\{(1-X) /(1+X)\}^{m / 2} \sum_{n=0}^{\infty}\{(1-X) / 2\}^{n}\left\{(-\alpha)_{n}(\alpha+1)_{n}\right\} /\left\{\Gamma(m+1)(1+m)_{n} n !\right\}
\end{aligned}
$$

where $(A)_{n}=A(A+1)(A+2) \cdots(A+n-1), \quad(A)_{0}=1$.

First, $P_{\alpha}^{0}(X)$ is calculated. From Eq. (21), $P^{0}{ }_{\alpha}(X)$ is written as

$$
\begin{aligned}
P_{\alpha}^{0}(X) & =\sum_{n=0}^{\infty}\{(1-X) / 2\}^{n}\left\{(-\alpha)_{n}(\alpha+1)_{n} / n !^{2}\right\} \\
& =\sum_{n=0}^{\infty}\{(1-X) / 2\}^{n}\left\{(-\alpha)(-\alpha+1) \cdots(-\alpha+n-1)(\alpha+1)(\alpha+2) \cdots(\alpha+n) / n !^{2}\right\} \\
& =1-\alpha \sum_{n=1}^{\infty}\{(1-X) / 2\}^{n}\left\{1 \cdot 2 \cdots(n-1) \cdot 1 \cdot 2 \cdots n / n !^{2}\right\}+O\left(\alpha^{2}\right) \\
& =1-\alpha \sum_{n=1}^{\infty}\{(1-X) / 2\}^{n} / n+O\left(\alpha^{2}\right) \\
& =1+\alpha \log \{1-(1-X) / 2\}+O\left(\alpha^{2}\right) \\
& =1+\alpha \log \{(1+X) / 2\}+O\left(\alpha^{2}\right)
\end{aligned}
$$

Therefore, for a sufficiently small positive $\alpha, P^{0}{ }_{\alpha}(\sin \theta)$ can be written as

$$
P_{\alpha}^{0}(\sin \theta)=1+\alpha \log \{(1+\sin \theta) / 2\}+O\left(\alpha^{2}\right)=1+2 \alpha \log \cos (\pi / 4-\theta / 2)+O\left(\alpha^{2}\right) .
$$

Next, $P^{m}{ }_{\alpha}(X)$ are calculated for $m \geqq 1$. From Eq. (21), $P^{m}{ }_{\alpha}(X)$ are written as

$$
\begin{aligned}
& P_{\alpha}^{m}(X)=(-1)^{m}\{\Gamma(\alpha+m+1) / \Gamma(\alpha-m+1)\}\{1 / \Gamma(m+1)\}\{(1-X) /(1+X)\}^{m / 2} \\
& \cdot \sum_{n=0}^{\infty}\left\{(-\alpha)_{n}(\alpha+1)_{n} /(1+m)_{n}\right\}(1 / n !)\{(1-X) / 2\}^{n} \\
&=(-1)^{m}\{(\alpha-m+1)(\alpha-m+2) \cdots(\alpha-1) \alpha / \Gamma(\alpha+1)\}\{\Gamma(\alpha+m+1) / \Gamma(m+1)\} \\
& \quad \cdot\{(1-X) /(1+X)\}^{m / 2} \sum_{n=0}^{\infty}\left\{(-\alpha)_{n}(\alpha+1)_{n} /(1+m)_{n}\right\}(1 / n !)\{(1-X) / 2\}^{n} \\
&=\alpha(-1)^{m}(-m+1)(-m+2) \cdots(-1)\{(1-X) /(1+X)\}^{m / 2}+O\left(\alpha^{2}\right) \\
&=-\alpha(m-1) !\{(1-X) /(1+X)\}^{m / 2}+O\left(\alpha^{2}\right) .
\end{aligned}
$$

Therefore, for a sufficiently small positive $\alpha, P^{m}{ }_{\alpha}(\sin \theta)$ can be written as 


$$
\begin{aligned}
P_{\alpha}^{m}(\sin \theta) & =-\alpha(m-1) !\{(1-\sin \theta) /(1+\sin \theta)\}^{m / 2}+O\left(\alpha^{2}\right) \\
& =-\alpha(m-1) ! \tan ^{m}(\pi / 4-\theta / 2)+O\left(\alpha^{2}\right) .
\end{aligned}
$$

From Eqs. (9), (22) and (23), one can obtain the following representation of the streamfunction $\Psi_{\text {in }}$ of the 87 modon for a sufficiently small positive $\alpha$ :

$$
\begin{aligned}
& \Psi^{M}{ }_{\text {in }}(\theta)= C_{\text {in }}-R U_{\text {in }} \sin \theta_{0} \cos \theta_{a}\left\{P^{0}{ }_{\alpha}(\sin \theta) / P^{1}{ }_{\alpha}\left(\sin \theta_{a}\right)+\sin \theta / \cos \theta_{a}\right\} \\
&= C_{\text {in }}+R^{2} \Omega\{1-\alpha(\alpha+1) / 2\} \sin \theta_{0} \cos \theta_{a} \\
&\left.\cdot\left[\left\{1+2 \alpha \log \cos (\pi / 4-\theta / 2)+O\left(\alpha^{2}\right)\right\} /\left\{-\alpha \tan { }_{\text {in }} \pi / 4-\theta_{a} / 2\right)+O\left(\alpha^{2}\right)\right\}+\sin \theta / \cos \theta_{a}\right] \\
&= C_{\text {in }}+R^{2} \Omega \sin \theta_{0} \cos \theta_{a} \\
& \cdot\left[-\{1+2 \alpha \log \cos (\pi / 4-\theta / 2)\} / \alpha \tan \left(\pi / 4-\theta_{a} / 2\right)+\sin \theta / \cos \theta_{a}\right]+O(\alpha) \\
&= C_{\text {in }}-\alpha^{-1} \cdot R^{2} \Omega \sin \theta_{0} \cos \theta_{a} / \tan \left(\pi / 4-\theta_{a} / 2\right) \\
& \quad-R^{2} \Omega \sin \theta_{0} \cos \theta_{a}\left\{2 \log \cos (\pi / 4-\theta / 2) / \tan (\pi / 4-\theta / 2)-\sin \theta / \cos \theta_{a}\right\}+O(\alpha) \\
&=\left\{\Psi^{M^{M}}(\theta) \text { of the } 90 \operatorname{modon}\right\}+O(\alpha),
\end{aligned}
$$

and

$$
\begin{aligned}
\Psi_{\text {in }}^{D_{\text {in }}}(\theta)= & -R U_{\text {in }} \cos \theta_{0} \cos \theta_{a}\left\{P^{1}{ }_{\alpha}(\sin \theta) / P^{1}{ }_{\alpha}\left(\sin \theta_{a}\right)-\cos \theta / \cos \theta_{a}\right\} \\
= & R^{2} \Omega\{1-\alpha(\alpha+1) / 2\} \cos \theta_{0} \cos \theta_{a} \\
& \quad \cdot\left[\left\{-\alpha \tan (\pi / 4-\theta / 2)+O\left(\alpha^{2}\right)\right\} /\left\{-\alpha \tan \left(\pi / 4-\theta_{a} / 2\right)+O\left(\alpha^{2}\right)\right\}-\cos \theta / \cos \theta_{a}\right] \\
= & R^{2} \Omega \cos \theta_{0} \cos \theta_{a}\left\{\tan (\pi / 4-\theta / 2) / \tan \left(\pi / 4-\theta_{a} / 2\right)-\cos \theta / \cos \theta_{a}\right\}+O(\alpha) \\
= & \left\{\Psi^{D}{ }_{\text {in }}(\theta) \text { of the } 90 \text { modon }\right\}+O(\alpha) .
\end{aligned}
$$

In Eq. (24), the constant terms

$$
C_{\text {in }}-\alpha^{-1} R^{2} \Omega \sin \theta_{0} \cos \theta_{a} / \tan \left(\pi / 4-\theta_{a} / 2\right)
$$

of the 87 modon corresponds to the constant term $C_{\text {in }}$ of the 90 modon. From Eqs. (10) and (17) one can easily see that the streamfunction $\Psi_{\text {ex }}$ of the 87 modon is expressed by the same equation as the 90 modon.

As for the potential vorticity $Q_{\text {in }}$ of the 87 modon, from Eqs. (7), (11), (18) and (24) the following representation for a sufficiently small positive $\alpha$ is obtained :

$$
\begin{aligned}
Q_{\text {in }} & =-k^{2}\left(\Psi_{\text {in }}-C_{\text {in }}\right) \\
& =-\left\{\alpha(\alpha+1) / R^{2}\right\}\left(\Psi_{\text {in }}-C_{\text {in }}\right)
\end{aligned}
$$

$$
\begin{aligned}
= & R^{-2}\left\{-R^{2} \Omega \sin \theta_{0} \cos \theta_{a}\right. \\
& \left.\quad / \tan \left(\pi / 4-\theta_{a} / 2\right)\right\}+O(\alpha) \\
= & \Omega \sin \theta_{0} \cos \theta_{a} / \tan \left(\pi / 4-\theta_{a} / 2\right)+O(\alpha) . \\
= & \bar{Q}_{\text {in }}+O(\alpha) \\
= & \left\{Q_{\text {in }} \text { of the } 90 \text { modon }\right\}+O(\alpha) .
\end{aligned}
$$

Finally as for Eq. (13) which is derived from the boundary conditions at $\theta=\theta_{a}$, using Eq. (23) one can easily obtain the following representation for sufficiently small positive $\alpha$ :

$$
\begin{aligned}
& \left(1 / U_{\mathrm{ex}}\right) P_{\beta}^{1}\left(-\sin \theta_{a}\right) / P_{\beta}^{2}\left(-\sin \theta_{a}\right) \\
& \quad=-\left(1 / U_{\mathrm{in}}\right) P_{\alpha}^{1}\left(\sin \theta_{a}\right) / P_{\alpha}^{2}\left(\sin \theta_{a}\right)
\end{aligned}
$$




$$
\begin{aligned}
& =-[-R \Omega\{1-\alpha(\alpha+1) / 2\}]^{-1} \\
& \qquad \cdot\left\{-\alpha(1-1) ! \tan \left(\pi / 4-\theta_{a} / 2\right)+O\left(\alpha^{2}\right)\right\} /\left\{-\alpha(2-1) ! \tan ^{2}\left(\pi / 4-\theta_{a} / 2\right)+O\left(\alpha^{2}\right)\right\} \\
& =(R \Omega)^{-1} \cot \left(\pi / 4-\theta_{a} / 2\right)+O(\alpha) \\
& =\{\text { the right hand side of Eq. }(20)\}+O(\alpha) .
\end{aligned}
$$

Eqs. (24), (25), (26) and (27) imply that the 87 modon changes continuously into the 90 modon as $\alpha \rightarrow 0$, that is, the set of the 87 modon with $\alpha$ $\rightarrow 0$ is included in the set of the 90 modon. Conversely, one can easily see that the set of the 90 modon is included in the set of the 87 modon with $\alpha \rightarrow 0$ (see Appendix).

Since Verkley (1990) showed that the 90 modon exists for the parameter values corresponding to the actual atmospheric situations, we can say that the 87 modon with a sufficiently small positive $\alpha$ exists for realistic parameter values.

\section{Stability properties}

Although the normal mode approach is a powerful method in studying the linear stability properties of steady basic states, the class of the basic states to which this method can be applied is extremely restricted. Modons do not belong to this class. Outside of this class the normal mode approach is applied numerically. The numerical approach commonly implies the replacement of an infinite-dimensional dynamical system by a finite dimensional one. But it is not easy to validate this replacement mathematically. Therefore even when a basic state is numerically shown to be linearly stable, the analytical confirmation is desired. On account of the fact that Verkley $(1987,1990)$ could not show the linear stability of the 87 and 90 modons numerically by applying the normal mode approach, the analytical investigation is still more required.

It is well known that a conserved quantity $A[\phi]$ called Arnol'd's invariant is a useful tool for analytical consideration of the stability of non-linear waves such as modons, to which the classical normal mode approach can not be applied analytically. But unfortunately
Arnol'd's invariant $A[\phi]$ which can be defined for the 87 modon does not exist for the 90 modon. Let us see this point more clearly.

First, let us briefly review the derivation of (linearized) Arnol'd's invariant $A[\phi]$. Let $\phi$ be any disturbance added to a prescribed stationary basic state streamfunction $\Psi$. Then the evolution equation of the total streamfunction $\Psi+\phi$ is given by

$$
\begin{aligned}
& \partial(Q+q) / \partial t+\mathrm{J}(\Psi+\phi, Q+q)=0, \\
& q=\Delta \phi .
\end{aligned}
$$

From Eq. (28), the linearized evolution equation of $\phi$ is obtained as

$$
\begin{aligned}
& \partial q / \partial t+\mathrm{J}(\Psi, q)+\mathrm{J}(\phi, Q)=0, \\
& q=\Delta \phi .
\end{aligned}
$$

Since $\Psi$ is a function of $Q$ because of the stationarity of $\Psi$, Eq. (29) can be rewritten as

$$
\partial q / \partial t+\mathrm{J}(Q,-\phi+q(d \Psi / d Q))=0 .
$$

Multiplying Eq. (30) by $-\phi+q(d \Psi / d Q)$ and integrating on the whole domain, one can easily obtain the following equation:

$$
\begin{aligned}
& \iint d \lambda d \sin \theta\{-\phi+q(d \Psi / d Q)\} \partial q / \partial t \\
& +\iint d \lambda d \sin \theta \mathrm{J}\left(Q,\{-\phi+q(d \Psi / d Q)\}^{2} / 2\right) \\
& =0 .
\end{aligned}
$$

From Eqs. (7) and (8), the values of $d \Psi / d Q$ of the 87 modon are given by

$$
\begin{array}{lll}
d \Psi / d Q=-1 / k^{2} & \text { for } & \pi / 2 \geqq \theta>\theta_{a}, \\
d \Psi / d Q=-1 / p^{2} & \text { for } & \theta_{a}>\theta \geqq \pi / 2 .
\end{array}
$$


Eqs. (32) and (33) imply that $-\phi+q(d \Psi / d Q)$ is discontinuous at $\theta=\theta_{a}$. Nevertheless the second integral of Eq. (31) vanishes because of the constancy of $Q$ at $\theta=\theta_{a}$. Then Eqs. (31), (32) and (33) give the following conservation law :

$$
\begin{aligned}
d A[\phi] / d t= & 0, \\
A[\phi]= & \iint d \lambda d \sin \theta(-\phi q) / 2 \\
& +\iint d \lambda d \sin \theta(d \Psi / d Q) q^{2} / 2, \\
q & =\Delta \phi .
\end{aligned}
$$

As mentioned above, this conservation law is a useful tool in studying the stability properties of the basic state $\Psi$.

As is clearly seen from the derivation of Eq. (34), $A[\phi]$ is well defined for the 87 modon. But unfortunately $A[\phi]$ does not exist for the 90 modon, because $Q=\bar{Q}_{\text {in }}=$ constant for $\pi / 2 \geqq \theta$ $>\theta_{a}$ and $d \Psi / d Q$ can not be defined there. This fact makes the analytical investigation of the stability properties of the 90 modon very difficult since there is no other general and powerful method comparable to Arnol'd's. But in section 3 it was shown that the 87 modon changes continuously into the 90 modon as $\alpha \rightarrow$ 0 , and so it might be conjectured that the stability properties of the 90 modon could be studied by means of $A[\phi]$ of the 87 modon for a sufficiently small positive $\alpha$. It is not so difficult to show this conjecture to be actually true. In the rest of this section, this matter is discussed.

Let $\Psi^{87}{ }_{\alpha}$ and $\Psi^{90}$ represent the streamfunction of the 87 and 90 modons, respectively. Then, the result of section 3 can be written as

$$
\lim _{\alpha \rightarrow 0} \Psi_{\alpha}^{87}=\Psi^{90} .
$$

Let it be supposed that Arnol'd's invariant $A[\phi]$ is proved negative definite for an arbitrary small positive $\alpha$. The negative definiteness of $A[\phi]$ is a sufficient condition for $\Psi^{87}{ }_{\alpha}$ to be linearly stable (e.g., see Benzi et al., 1982; Laedke and Spatschek, 1986). If the disturbance $\phi$ is written as

$$
\phi(\lambda, \theta, t)=\operatorname{Re}\left\{\bar{\phi}(\lambda, \theta) e^{\sigma t}\right\},
$$

then $\bar{\phi}$ and $\sigma$ must satisfy the following equation because $\phi$ is a solution to Eq. (29) :

$$
\begin{aligned}
\sigma \Delta \bar{\phi} & +\mathrm{J}\left(\Psi^{87}{ }_{\alpha}, \Delta \bar{\phi}\right) \\
& +\mathrm{J}\left(\Delta \Psi^{87}{ }_{\alpha}+2 \Omega \mu, \bar{\phi}\right)=0 .
\end{aligned}
$$

Since $\Psi^{87}{ }_{\alpha}$ is linearly stable, for any eigenvalue $\sigma=\sigma_{\alpha}$ the real part of $\sigma_{\alpha}$ is not positive,

$$
\operatorname{Re}\left\{\sigma_{\alpha}\right\} \leqq 0 \quad \text { for any } \sigma_{\alpha} .
$$

Eq. (38) implies that the following equation must hold, because this equation is valid for an arbitrary small $\alpha$ :

$$
\lim _{\alpha \rightarrow 0} \operatorname{Re}\left\{\sigma_{\alpha}\right\} \leqq 0 \quad \text { for any } \sigma_{\alpha} .
$$

Taking the limit $\alpha \rightarrow 0$ of Eq. (37) and using the facts

$$
\begin{gathered}
\lim _{\alpha \rightarrow 0} \mathrm{~J}\left(\Psi_{\alpha}^{87}{ }_{\alpha}, \Delta \phi\right)=\mathrm{J}\left(\lim _{\alpha \rightarrow 0} \Psi_{\alpha}^{87}, \Delta \phi\right), \\
\lim _{\alpha \rightarrow 0} \mathrm{~J}\left(\Delta \Psi_{\alpha}^{87}+2 \Omega \mu, \phi\right) \\
=\mathrm{J}\left(\lim _{\alpha \rightarrow 0} \Delta \Psi^{87}{ }_{\alpha}+2 \Omega \mu, \phi\right) \\
=\mathrm{J}\left(\Delta \lim _{\alpha \rightarrow 0} \Psi_{\alpha}^{87}+2 \Omega \mu, \phi\right),
\end{gathered}
$$

together with Eq. (35), it is easy to see that $\lim _{\alpha \rightarrow 0} \sigma_{\alpha}$ are eigenvalues for the 90 modon. Therefore Eq. (39) implies the linear stability of the 90 modon.

The above argument reveals that the negative definiteness of Arnol'd's invariant $A[\phi]$ of the 87 modon for an arbitrary small positive $\alpha$ is a sufficient condition for the linear stability of the 90 modon.

\section{Conclusion}

In this note, a relation between the 90 and 87 modons was investigated. As a result, the 90 modon was shown to be a limiting case of the 87 modon as $\alpha \rightarrow 0$. Thereby it was revealed that the linear stability of the 87 modon by Arnol'd's invariant $A[\phi]$ for an arbitrary small $\alpha$ implies the linear stability of the 90 modon. Therefore it is sufficient for the linear stability of the 90 modon that Arnol'd's invariant $A[\phi]$ of the 87 modon is demonstrated to be negative definite for an arbitrary small positive $\alpha$. This 
demonstration belongs to future study.

\section{Appendix}

The boundary condition equation for the 87 modon is given by

$$
\begin{aligned}
& \left(1 / U_{\mathrm{ex}}\right) P_{\beta}^{1}\left(-\sin \theta_{a}\right) / P_{\beta}^{2}\left(-\sin \theta_{a}\right) \\
& =-\left(1 / U_{\mathrm{in}}\right) P^{1}{ }_{\alpha}\left(\sin \theta_{a}\right) / P^{2}{ }_{\alpha}\left(\sin \theta_{a}\right),
\end{aligned}
$$

where

$$
\alpha(\alpha+1)=2\left(R \Omega / U_{\text {in }}+1\right)=R^{2} k^{2}, \quad \alpha>-1 / 2,
$$

and

$$
\beta(\beta+1)=2\left(R \Omega / U_{\mathrm{ex}}+1\right)=R^{2} p^{2}, \quad \beta>-1 / 2 .
$$

Let $\theta^{m}{ }_{\beta}$ and $\bar{\theta}^{m}{ }_{\beta}$ be the first and second greatest zeros of $P^{m}{ }_{\beta}(-\sin \theta)$ for $-\pi / 2<\theta<\pi / 2$,

then $\bar{\theta}^{1}{ }_{\beta}<\theta^{2}{ }_{\beta}<\theta^{1}{ }_{\beta}$,

and $\operatorname{sign}\left\{P_{\beta}^{1}(-\sin \theta)\right\}=\operatorname{sign}\left\{P_{\beta}^{2}(-\sin \theta)\right\}$

$$
\text { for } \theta^{1}{ }_{\beta}<\theta<\pi / 2 \text {. }
$$

Zeros approach $\pi / 2$ as $\beta$ decreases, and they disappear one by one as $\beta$ decreases beynod each integer. From Eq. $(A-2)$, the function in the left hand side of Eq. $(A-1)$ behaves as follows (see Fig. 3) :

$$
F(\theta, \beta)>0 \quad \text { for } \quad \bar{\theta}^{1}{ }_{\beta}<\theta<\theta^{2}{ }_{\beta},
$$

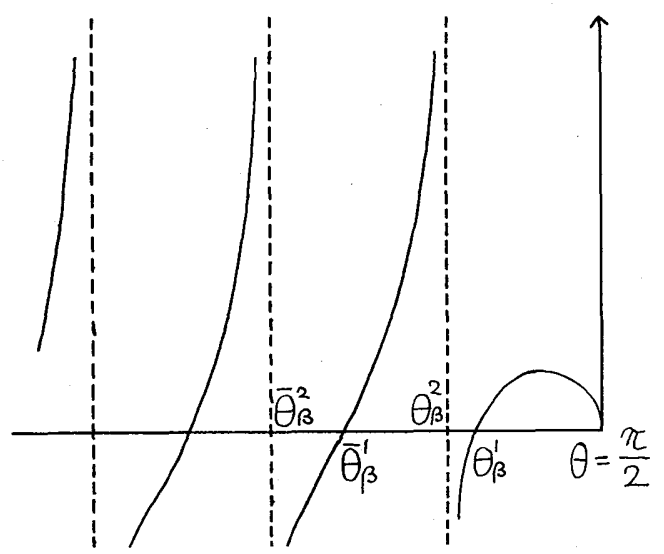

Fig. 3 The graph of $F(\theta, \beta)$.

$$
\begin{array}{lll}
F(\theta, \beta) \rightarrow 0 & \text { as } \theta \rightarrow \bar{\theta}^{1}{ }_{\beta} & \text { from above, } \\
F(\theta, \beta) \rightarrow \infty & \text { as } \theta \rightarrow \theta^{2}{ }_{\beta} & \text { from below, }
\end{array}
$$

where

$$
F(\theta, \beta)=\left(1 / U_{\mathrm{ex}}\right) P_{\beta}^{1}(-\sin \theta) / P^{2}{ }_{\beta}(-\sin \theta) .
$$

In Fig. $3, \bar{\theta}^{1}{ }_{9} \leqq \bar{\theta}^{1}{ }_{\beta} \leqq \theta^{1}{ }_{8}$ and $\theta^{2}{ }_{9} \leqq \theta^{2}{ }_{\beta} \leqq \pi / 2$ for 9 $\geqq \beta \geqq 8$, where $\bar{\theta}^{1}{ }_{9} \simeq 47.6^{\circ}, \theta^{1}{ }_{8} \simeq 64.2^{\circ}$ and $\theta^{2}{ }_{9} \simeq$ $58.8^{\circ}$. $\beta=8(=9)$ corresponds to $U_{\text {ex }} \cos 45^{\circ}=$ $9.4 \mathrm{~m} / \mathrm{s}(=7.4 \mathrm{~m} / \mathrm{s})$.

On the other hand, for $0 \leqq \alpha<1$, the function in the right hand side of Eq. $(\mathrm{A}-1)$ behaves as follows (see Fig. 4) :

$$
G(\theta, \alpha)>0 \text { and monotonically increasing }
$$

$$
\begin{array}{lll} 
& \text { for } \quad-\pi / 2<\theta<\pi / 2, \\
G(\theta, \alpha) \rightarrow \infty \quad \text { as } \quad \theta \rightarrow \pi / 2, \\
G(\theta, \alpha) \rightarrow 0 \quad \text { as } \quad \theta \rightarrow-\pi / 2, \quad(\mathrm{~A}-4)
\end{array}
$$

where

$$
G(\theta, \alpha)=-\left(1 / U_{\text {in }}\right) P_{\alpha}^{1}(\sin \theta) / P^{2}(\sin \theta) .
$$

As demonstrated in section $3, G(\theta, \alpha)$ approaches the function in the right hand side of the boundary condition Eq. (20) for the 90 modon, that is,

$$
G(\theta, \alpha) \rightarrow(1 / R \Omega) \cot (\pi / 4-\theta / 2)
$$

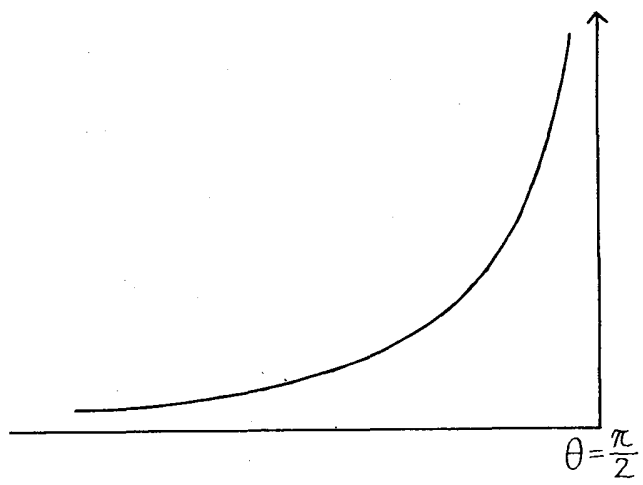

Fig. 4 The graph of $G(\theta, \alpha)$. 


$$
\text { as } \alpha \rightarrow 0 . \quad(\mathrm{A}-5)
$$

From Fig. 3 and Fig. 4, we can see that the graphs of $F(\theta, \beta)$ and $G(\theta, \alpha)$ have at least one intersection (multiple intersections in general). In other words, there exists at least one pair of $\beta$ and $\theta_{a}$ for any $\alpha \in[0,1)$ such that Eq. $(\mathrm{A}-1)$ is satisfied. Together with Eq. $(\mathrm{A}-5)$, this implies that

$\lim _{\alpha \rightarrow 0}$ the set of the 87 modons

$$
=\text { the set of the } 90 \text { modons. } \quad(\mathrm{A}-6)
$$

\section{References}

Benzi, R., S. Pierini, E. Salusti and A. Vulpiani, 1982: On nonlinear hydrodynamic stability of planetary vortices. Geophys. Astrophys. Fluid Dyn., 20, 293-306.

Butchart, N., K. Haines and J. C. Marshall, 1987 : A theoretical and diagnostic study of solitary waves and atmospheric blocking. J. Atmos. Sci., 46, 2063-2078.

Haines, K. and J. C. Marshall, 1987 : Eddy forced coherent structures as a prototype of atmospheric blocking. Quart. J. R. Meteor.
Soc., 113, 681-704.

Laedke, E. W. and K. H. Spatschek, 1986: Two dimensional drift vortices and their stability. Phys. Fluids, 29, 133-142.

Larichev, V. D. and G. M. Reznik, 1976: Two dimensional Rossby soliton: An exact solution. POLYMODE News, No. 19, 3 and 6.

McIntyre, M. E. and T. G. Shepherd, 1987: An exact local conservation theorem for finite amplitude disturbances to non parallel shear flows, with remarks of Hamiltonian structure and on Arnol'd's stability theorem. J. Fluid Mech. 181, 527-565.

McWilliams, J. C., 1980 : An application of equivalent modons to atmospheric blocking. Dyn. Atmos. Oceans, 5, 43-66.

Stern, M. E., 1975 : Minimal properties of planetary eddies. J. Mar. Res., 33, 1-13.

Verkley, W. T. M., 1984: The construction of barotropic modons on a sphere. J. Atmos. Sci., 41, 2492-2504.

Verkley, W. T. M., 1987 : Stationary barotropic modons in westerly background flows. $J$. Atmos. Sci., 44, 2383-2398.

Verkley, W. T. M., 1990 : Modons with uniform absolute vorticity. J. Atmos. Sci., 47, 727-745.

\title{
Verkley の 1987 年モドンと 1990 年モドンの関係
}

\author{
金久博忠
}

1987 年と 1990 年に Verkley は，大気ブロッキング現象のモデルの一つとして球面上のモドンを構成した (以 下, 87 モドン及び 90 モドンと言う)。これら二つのモドンは球面上の順圧渦位方程式の厳密解として独立に提出 された。この報告では, 87 モドンに含まれている助変数 $k^{2}$ が零に近づく時, 87 モドンは連続的に 90 モドンへ変 化する事が示される。ここで助変数 $k^{2}$ は，モドン内部領域に於ける $-d Q / d \Psi$ の值であり, $Q$ は渦位, $\Psi$ は流線 関数である。

定常解の安定性を調べるのにアーノルド不変量が有用である事は良く知られている。アーノルド不変量は 87 モドンに対しては存在するが, 90 モドンに対しては定義されない。しかし, 90 モドンが 87 モドンの $k^{2} \rightarrow 0$ の極 限である事により，十分小さな $k^{2}$ に対する 97 モドンのアーノルド不変量を使って, 90 モドンの安定性を調べる 事が可能となる事が示される。 